Published in final edited form as:

Gastroenterology. 2019 May ; 156(6): 1617-1626.e1. doi:10.1053/j.gastro.2019.01.253.

\title{
DEVELOPMENT AND VALIDATION OF A MUCOSAL IMPEDANCE CONTOUR ANALYSIS SYSTEM TO DISTINGUISH ESOPHAGEAL DISORDERS
}

\author{
Dhyanesh A. Patel, MD1, Tina Higginbotham, MPA ${ }^{1}$, James C. Slaughter, DrPH², \\ Muhammad Aslam, MD ${ }^{1}$, Elif Yuksel, MD ${ }^{3}$, David Katzka, MD ${ }^{4}$, C. Prakash Gyawali, MD, \\ MRCP5, Melina Mashi, PhD ${ }^{6}$, John Pandolfino, MD ${ }^{6}$, Michael F. Vaezi, MD, PhD ${ }^{1}$ \\ ${ }^{1}$ Division of Gastroenterology, Hepatology and Nutrition, Vanderbilt University Medical Center. \\ ${ }^{2}$ Department of Biostatistics, Vanderbilt University Medical Center. \\ ${ }^{3}$ Department of Gastroenterology, Izmir Ataturk Teaching and Research Hospital, Katip Celebi \\ University, Izmir, Turkey. \\ ${ }^{4}$ Division of Gastroenterology and Hepatology, Mayo Clinic, Rochester, MN. \\ ${ }^{5}$ Division of Gastroenterology, Washington University Medical Center, St. Louis, MO. \\ ${ }^{6}$ Division of Gastroenterology and Hepatology, Northwestern University Feinberg School of \\ Medicine, Chicago, Illinois.
}

\begin{abstract}
Background: Diagnostic testing for chronic esophageal disorders rely on histopathology analysis of biopsies or uncomfortable transnasal catheters or wireless $\mathrm{pH}$ monitoring, which capture abnormal intraluminal refluxate. We therefore developed a balloon mucosal impedance (MI) catheter system that instantly detects changes in esophageal mucosal integrity during endoscopy over a long segment of the esophagus. We performed a prospective study to evaluate the ability of a balloon-incorporated MI catheter to detect and evaluate esophageal disorders, including gastroesophageal reflux disease (GERD) and eosinophilic esophagitis (EoE).
\end{abstract}

Methods: We performed a prospective study of 69 patients undergoing esophagogastroduodenoscopy with or without wireless $\mathrm{pH}$ monitoring. Patients were classified as having GERD (erosive esophagitis or abnormal $\mathrm{pH} ; \mathrm{n}=24$ ), EoE (confirmed with pathology

\footnotetext{
Correspondence: Michael F. Vaezi, MD, PhD, MSc (Epi), FACG, Professor of Medicine, Clinical Director, Division of Gastroenterology, Hepatology and Nutrition, Director, Center for Swallowing and Esophageal Disorders, Vanderbilt University Medical Center, Nashville, TN, 615-322-3739 (phone), 615-322-8285 (fax), Michael.vaezi@ vumc.org.

Author contributions: Dhyanesh Patel (acquisition of data, analysis and interpretation of data, drafting of the manuscript, critical revision of the manuscript), Tina Higginbotham (acquisition of data), James Slaughter (analysis of the data), Muhammad Aslam (study concept), Elif Yuksel (study concept), David Katzka (acquisition of external data), C. Prakash Gyawali (acquisition of external data), John Pandolfino (acquisition of external data), and Michael Vaezi (study concept and design, acquisition of data, analysis and interpretation of data, critical revision of the manuscript, study supervision).

Disclosures and Conflicts of Interest: Vanderbilt University and Diversatek Healthcare Inc. (Denver, CO, USA) jointly hold a patent on the mucosal impedance (MI) concept and device. This was disclosed to patients. MV, DK, CPG, and JP have had research funding from Diversatek Healthcare in the conduct of studies with mucosal impedance. Diversatek Healthcare had no influence on the study design, conduct, analysis or the final manuscript. There are no financial relationships between any of the other authors and Diversatek Healthcare Inc. All other authors (DP, TH, JC, MA, MM, EY) have no conflicts of interest.
} 
analysis of tissues from both distal and proximal esophagus; $\mathrm{n}=21$ ), or non-GERD (normal results from esophagogastroduodenoscopy and $\mathrm{pH}$ tests; $\mathrm{n}=24$ ). Receiver operating characteristic curves (ROC) and area under the ROC curve (AUC) were used to compare the accuracy of balloon MI in diagnosis. Probabilities of assignment to each group (GERD, non-GERD, or EoE) were estimated using multinomial logistic regression. Association between MI patterns and diagnoses were validated using data from patients seen at 3 separate institutions.

Results: MI pattern along the esophageal axis differed significantly $(p<0.01)$ among patients with GERD, EoE and non-GERD. Patients with non-GERD had higher MI values along all measured segments. The MI pattern for GERD was easily distinguished from that of EoE: in patients with GERD, MI values were low in the distal esophagus and normalized along the proximal esophagus, whereas in patients with EoE, measurements were low in all segments of the esophagus. Intercept and rate of rise of MI value (slope) as distance increased from the squamocolumnar junction identified patients with GERD with an $\mathrm{AUC}=0.69$, patients with EoE with an AUC of 0.89, and patients with non-GERD with an AUC $=0.84$ in the development cohort. One patient had an adverse event (reported mild chest pain after the procedure) and was discharged from the hospital without further events.

Conclusions: We developed a balloon MI catheter system that instantly detects changes in esophageal mucosal integrity during endoscopy and found it to be safe and able to identify patients with GERD, EoE, or non-GERD. We validated our findings in a separate cohort for patients.

\section{Clinicaltrials.gov:}

\section{Keywords}

diagnostic; prognostic; predictive model; impedance contour

\section{INTRODUCTION}

Gastroesophageal reflux disease (GERD) is the leading outpatient physician diagnosis for gastrointestinal disorders in the United States affecting approximately $18-27 \%$ of the population. ${ }^{1-3}$ Despite the high prevalence and projected national expenditures ranging from $\$ 9.3$ to $\$ 12.1$ billion, ${ }^{4,5}$ testing for GERD has primarily relied on either presence of endoscopic esophagitis or demonstration of abnormal esophageal acid exposure during an ambulatory $\mathrm{pH}$ or intraluminal impedance monitoring. However, it is well known that transnasal catheter-based testing is associated with significant patient discomfort (nose pain, throat pain, cough, chest discomfort), which can lead to alteration in patient's daily activities (less likely to be active, more likely to skip meals, and have difficulty with swallowing) leading to variable sensitivity of these tests. ${ }^{6,7}$ Wireless ambulatory $\mathrm{pH}$ monitoring is associated with less patient discomfort, however, it only measured the acidity of refluxed material at a single point along the esophagus (6-cm above squamo-columnar junction, SCJ) and can be subject to early detachment in $10 \%$ of examinations leading to extra cost burden of repeat testing and more importantly, delay in diagnosis for the patient. ${ }^{8}$ Furthermore, these tests fail to account for day to day variability of reflux as they only provide 24 to 48 hour snap shot of a disease process that is chronic in nature. ${ }^{9}$ Thus, current diagnostic testing 
in GERD are suboptimal and can have false negative rate of nearly $30 \%$ in patients that have endoscopic esophagitis. ${ }^{10,11}$

Eosinophilic esophagitis (EoE) is another esophageal disease that is increasing in prevalence and estimated to affect 57 per $100,000 .{ }^{12}$ EoE is an chronic immune mediated disease characterized by symptoms of esophageal dysfunction and histologically by eosinophilpredominant inflammation in the esophagus. ${ }^{13}$ Diagnosis is made using endoscopy and distal and proximal esophageal biopsies showing at least 15 eosinophils per high-power field $(\mathrm{HpF})$. There is also significant overlay between GERD and EoE, with both often in the differential especially for young patients with dysphagia, which can lead to separate testing for each to determine the etiology.

Direct measurement of esophageal epithelial integrity employing mucosal impedance (MI) has the potential to obviate the need for repeat endoscopies in EoE and short term prolonged $\mathrm{pH}$ monitoring for GERD. ${ }^{14}$ Patients with GERD or EoE have dilation of intercellular spaces (DIS) between esophageal epithelial cells (termed spongiosis), which are regulated by tight junction proteins. ${ }^{15-17}$ The degree of DIS is shown to inversely correlate with MI measurements in adult ${ }^{18}$ and pediatric ${ }^{19}$ population showing that MI could serve as a surrogate marker of histological changes in these patients. In a proof of concept study, we have previously shown that MI pattern along the esophageal axis is significantly different in patients with GERD (erosive or non-erosive reflux disease) or EoE compared to patients without GERD or patients with achalasia. ${ }^{14}$ MI pattern also had higher specificity $(95 \%)$ and positive predictive values (96\%) compared to wireless $\mathrm{pH}$ monitoring (64\% and $40 \%$, respectively) for identifying patients with esophagitis. ${ }^{14}$ Furthermore, in a prospective, double-blinded study using MI pattern along the esophageal axis, MI accurately predicted EoE during endoscopy with a sensitivity of $100 \%$ and specificity of $96 \%$ without the need for histology. ${ }^{20} \mathrm{MI}$ can also be used to monitor treatment response with normalization of values post-therapy. ${ }^{14}$

However, a single channel MI catheter, employed in the proof of concept studies outlined above, might be subject to increased inter-provider variability due to lack of adequate contact with mucosa from catheter movement and intraluminal gas and fluid (due to $360^{\circ}$ design of the impedance sensors). ${ }^{21}$ This catheter also makes point measurements which may not accurately assess the patchy distribution of epithelial change in EoE. Thus, we developed a new balloon MI catheter system to further diminish measurement variability by incorporating both radial and axial sensors mounted on a balloon to measure esophageal mucosal integrity at 180 degree intervals along a $10-\mathrm{cm}$ segment of the esophagus. The aims of this prospective multi-center study were to 1) investigate balloon MI patterns in patients with GERD and EoE and 2) develop a prediction model using MI measurements that can be used by physicians to reliably and rapidly distinguish various esophageal pathology (GERD, EoE, or non-GERD) during endoscopy.

\section{PATIENTS AND METHODS}

The study was performed in accordance with the Declaration of Helsinki, Good Clinical Practice, and applicable regulatory requirements. Each patient signed a consent form before 
undergoing any study-related procedures. The Vanderbilt Institutional Review Board approved this clinical trial (IRB \# 140470). All authors had access to the study data and reviewed and approved the final manuscript.

\section{Study design and patient population:}

The study population consisted of patients referred for evaluation of GERD or EoE at the Esophageal Motility Center at Vanderbilt University Medical Center or the Mayo Clinic. Patients referred for evaluation of GERD underwent endoscopy as well as ambulatory wireless 48 hour $\mathrm{pH}$ monitoring one week off acid suppressive treatment. Presenting symptoms in this group included typical symptoms of GERD (heartburn, regurgitation) or atypical symptoms (epigastric pain, chronic cough, hoarseness, and asthma) with both groups having an incomplete response to acid suppressive therapy. The external validation cohort obtained from Mayo Clinic, Washington University in St. Louis, and Northwestern University used this same inclusion and exclusion criteria. The following information were collected from all patients: demographic data (age, sex, race, body mass index), presence of GERD symptoms (heartburn with or without regurgitation), extra-esophageal symptoms (cough, hoarseness, epigastric/chest pain or asthma), or dysphagia. All patients with dysphagia had distal and proximal esophageal biopsies to evaluate for eosinophilic esophagitis and underwent high-resolution manometry to exclude motility disorder. The presence and size of a hiatal hernia along with the presence and severity of esophagitis (graded by the Los Angeles Classification: A, B, C, or D) were determined with endoscopy.

${ }^{22}$ In patients with EoE, the diagnosis was confirmed by pathology (> 15 eosinophils/HpF in both distal and proximal esophageal biopsies). The primary presenting symptoms in this group was dysphagia who were not previously treated with PPI's, steroid therapy or dietary intervention. Wireless $\mathrm{pH}$ testing was not conducted in this group as it is not included in the current recommendations for initial evaluation of EoE. ${ }^{23}$ Patients with age below 18 years or history of radiation, malignancy, recent esophageal surgery, known Barrett's esophagus, or narrow caliber esophagus that precluded safe expansion of the balloon MI were not approached for this study. All patients underwent esophagogastro-duodenoscopy (EGD). Based on endoscopic, $\mathrm{pH}$ monitoring and pathology findings, patients were stratified into three groups: 1) GERD [erosive esophagitis or abnormal $\mathrm{pH}$ (total percent time $\mathrm{pH}<4$ of $>5.5 \%], 2$ ) EoE, and 3) Non-GERD (normal EGD and pH). All patients in the study underwent the balloon MI testing during endoscopy and prior to deployment of wireless $\mathrm{pH}$ capsule or esophageal biopsies.

\section{Balloon Mucosal Impedance (MI):}

A newly designed balloon MI catheter (Figure 1) was engineered to measure electrical impedance of the esophageal lining by direct mucosal contact (Diversatek Healthcare Inc, Denver, CO). Special sensor columns designed to detect changes in mucosal impedance were mounted on a balloon made from medical grade Polyethylene Terephthalate (PET), which is specifically used for its non-compliant properties that prevent expansion beyond 2 $\mathrm{cms}$ in diameter. The catheter body is manufactured from medical grade PolyEther Blok Amide (PEBAX), which is routinely used in esophageal catheters and is biocompatible. The sensors were spaced at $1 \mathrm{~cm}$. The initial design included four axial columns of 9 impedance channels (total of 36) separated by 90 degree intervals with length of $10 \mathrm{~cm}$ mounted on an 
inflatable balloon (Figure 1). The most recent modification included reduction to two axial columns of 9 channels (total of 18) separated by 180 degree intervals on the balloon. Most patients (75\%) underwent testing using four columns balloon MI catheter, while $25 \%$ of patients underwent testing using the two columns balloon MI catheter. The two columns balloon MI catheter is the newest modification of the device as we have previously shown that radial impedance (measurement of MI at 90 degree intervals) did not add any diagnostic utility compared to axial pattern of MI along the esophageal axis in patients with EoE (supplemental Figure 1A) or GERD (supplemental Figure 1B). ${ }^{24}$ MI balloon was introduced into the esophagus through the mouth under endoscopic guidance with the most distal sensors just above the SCJ. Direct contact of the MI sensors with the esophageal epithelium was obtained by inflating an intra-esophageal balloon assembly in a controlled fashion using a calibrated inflation device (Boston Scientific, Marlsborough, MA). The balloon assembly is attached to a channeled feed that records axial MI measurements (in ohms, $\Omega$ ) on a $10 \mathrm{~cm}$ segment of the esophagus with radial measurements from sensors at 2 or 4 columns over 90 seconds. Data is displayed in a tabular and heat map format (contour of esophageal mucosal integrity) on a dedicated computer using a software program designed by Diversatek Healthcare Inc. The frequency for the measuring circuit was set at $10 \mathrm{~Hz}$. Once the MI data was recorded, the balloon assembly was deflated and then removed by the provider. The overall procedure added approximately $2-3$ minutes of additional procedure time for each patient.

\section{Wireless pH Monitoring:}

All patients who were referred for diagnostic testing for GERD underwent 48 hour ambulatory wireless $\mathrm{pH}$ monitoring (Given Imaging, Duluth, GA). They were instructed to stop taking all proton pump inhibitors and $\mathrm{H} 2$-receptor antagonists for at least 7 days prior to undergoing ambulatory $\mathrm{pH}$ monitoring. The wireless device was deployed during upper endoscopy after visual anatomic inspection and distance measurements from the incisors to the SCJ. Wireless capsules were calibrated by submersion in buffer solutions at $\mathrm{pH} 7.0$ and $\mathrm{pH} 1.0$, and then activated by magnet removal. They were placed using the manufacturer's delivery system at $6 \mathrm{~cm}$ above the SCJ and attached with vacuum suction as previously described. ${ }^{25}$ Capsule placement was confirmed with endoscopy. Subsequently, patients were given wireless $\mathrm{pH}$ recorders to wear on their waists, or to keep within 3 feet to 5 feet at all times. Recording devices receive $\mathrm{pH}$ data sampling transmitted by the capsule at $433 \mathrm{~Hz}$ with 6 second sampling intervals and $\mathrm{pH}$ recording is conducted for a total of 48 hours. Patients were instructed to perform their normal daily activities and dietary practices. ${ }^{26}$ Measurements of the total, upright, supine percentage time when esophageal $\mathrm{pH}$ was below 4 were determined over day 1 and day 2 of the wireless study. Acid exposure time (percent time $\mathrm{pH}$ was $<4$ ) of greater than $5.5 \%$ per day was considered abnormal. ${ }^{8}$

\section{Statistical Analysis:}

Data were collected and stored at the secure Web based Vanderbilt Digestive Disease Center REDCap (Research Electronic Data Capture) (1 UL1 RR024975 NCRR/NIH). MI data were processed for each subject in the following steps. First, MI values were set to missing if we observed no variability in a column over distance, a MI value was over 12000, or a studentized residual was above 3.5. We then calculated the MI at each distance by averaging 
across the non-missing channels for each distance. For the 2- and 4- column catheter, we averaged over at most 2 and 4 values, respectively, at each distance. Cleaning steps were evaluated for appropriateness prior to considering outcome models. In addition to using the MI at each distance, we also summarized the MI using an intercept and slope. The intercept and slope were calculated by conducting separate simple linear regressions by subject using MI (outcome) and distance (continuous predictor) to estimate the intercept and slope parameter. The intercept and slope thus correspond to the proximal MI measurement and change in MI over time. Sensitivity analysis were also conducted. There was strict control and supervision of the data entry and access for this study.

Patient characteristics were described using medians and interquartile ranges (IQR, 25 $5^{\text {th }}$ and $75^{\text {th }}$ percentiles) for continuous variables and proportions for categorical variables.

Statistical differences in outcomes among groups and various esophageal sites were assessed using Kruskal-Wallis test, Pearson $X^{2}$ test, or the Wilcoxon test. $P<0.05$ was considered significant. Receiver operating characteristic (ROC) curves and area under the ROC curve (AUC) were used to compare predictive accuracy of balloon MI for GERD, EoE, and nonGERD subjects. Separate multivariable logistic regression models were fit employing either GERD (vs EoE and non-GERD), EoE (versus GERD and non-GERD) or non-GERD (versus GERD and EoE) as the outcome of interest. We used two-predictor models with the predictors being either continuous measures of (1) MI at $2 \mathrm{~cm}$ and MI at $5 \mathrm{~cm},(2) \mathrm{MI}$ at $2 \mathrm{~cm}$ and MI at $10 \mathrm{~cm}$, and (3) intercept and slope of MI (defined above). The intercept and slope model predictors gave consistently good results across settings and efficiently use all of the MI data across distance, so we focus on the results from these models. Predicted probabilities of diagnosis group (GERD, non-GERD, or EoE) as a function of MI intercept and MI slope were estimated using multinomial logistic regression. This model assumes that subjects must belong to one of the three diagnosis groups, and uses the baseline prevalence of GERD, non-GERD, and EoE $(35 \%, 35 \%, 30 \%)$ to estimate conditional probability of group membership given MI intercept and slope.

Sample size, or allowable model complexity, was based primarily on the consideration that a model must fit no more than $\mathrm{m} / 10$ to $\mathrm{m} / 15$ parameters to be reliable and reproducible on future similar samples. For categorical outcomes as in our study, $\mathrm{m}$ is the number of subjects in each disease group ( $\mathrm{m}$ ranged from 21 to 24 in our sample), so we were a priori limited to considering at most two potential predictors. To mitigate overfitting, we pre-specified three possible models with only two predictors each: MI at $2 \mathrm{~cm}$ and MI at $5 \mathrm{~cm}$, MI at $2 \mathrm{~cm}$ and $\mathrm{MI}$ at $10 \mathrm{~cm}$, and the MI intercept and slope. The intercept and slope approach was based on data reduction techniques that allow us to use all of the MI data but limit the degrees of freedom in the predictive model to comply with the allowable model complexity. Our primary power consideration demonstrates sufficient sample size to build and validate 2predictors models. For continuous predictors, we will have atleast $80 \%$ power to detect an odds ratio of 2.2 per standard deviation increase in the predictors. All calculations assume a 2 -sided significance level of 0.05 . We subsequently validated the pattern of mucosal impedance contour for GERD and EoE using an external data set from three tertiary care centers (Mayo Clinic, Washington University in St. Louis, and Northwestern University). 


\section{RESULTS}

\section{Demographics:}

A total of 69 patients were studied and stratified into those with GERD ( $\mathrm{n}=24)$, non-GERD $(\mathrm{n}=24)$ and $\operatorname{EoE}(\mathrm{n}=21)$ (Table 1). Overall, 83\% of patients with GERD had esophagitis (LA grade A, 20\%; grade B, $40 \%$; grade C, $30 \%$; and grade D, $10 \%$ ) with $27 \%$ of them having a hiatal hernia. As expected, patients with EoE were younger with median (IQR) age of 33 years (26-38) and more likely to present with dysphagia as the primary complaint (93\%) compared to non-GERD or patients with GERD $(p<0.01)$. The median (IQR) eosinophilic count/hpf was 50 (30-100) in the proximal and 47 (40-50) in the distal esophagus. As expected, patients with GERD had significantly higher acid exposure time (total, upright, and supine \%time $\mathrm{pH}$ was $<4)$ compared to those without GERD $(p<0.01)$. The groups were otherwise similar.

\section{Balloon MI}

Group differentiation-—Overall, median (IQR) MI measurements $(\Omega)$ were significantly $(p<0.01)$ lower for GERD and EoE groups at all esophageal sites compared to non-GERD subjects (Table 2, Figure 2A). In GERD, MI measurements were lower in the distal esophagus (closer to $\mathrm{SCJ}$ ) and had incremental rise in MI values proximally away from the SCJ (Figure 2A); patients with EoE had even lower baseline MI values with only small incremental increase in MI values from distal to proximal esophagus. Non-GERD subjects had higher baseline MI values in the distal esophagus and MI values remained elevated along the esophagus. The three groups displayed unique esophageal mucosal contour displayed as a colorcoded MI values as a function of disease state and distance along the esophagus (Figure 2B). This contour heatmap could easily be employed to establish a diagnosis during endoscopy independent of biopsy or $\mathrm{pH}$ monitoring.

We validated our esophageal mucosal contour for GERD and eosinophilic esophagitis in an external cohort from three tertiary care centers (Mayo Clinic, Washington University in St. Louis, and Northwestern University). The cohort included 28 patients with GERD (68\% female, $89 \%$ Caucasian) defined as presence of esophagitis or total $\%$ time $\mathrm{pH}<4$ of $>5.5 \%$. Our cohort of patients with eosinophilic esophagitis also included 8 patients from Mayo Clinic, which we have plotted separately to demonstrate that esophageal mucosal contour is similar between internal and external cohorts as shown in Figure 2A.

Since the three groups varied based on initial MI values in distal esophagus (intercept) with increasing values proximally (slope), discrimination of the three groups were tested based on pre-specified location along the esophagus (2- and 5-cm as well as 2- and 10-cm from the SCJ) as well as MI values based on the intercept and slope (Table 3). The best fit in predicting the three groups were achieved by employing intercept and slope (Table 3). Receiver operating characteristic analysis of intercept and slope of balloon MI measurements reliably predicted GERD (AUC $=0.69$ ), EoE (AUC $=0.89$ ), or non-GERD $($ AUC $=0.84)$ (Figure 3). Based on esophageal MI intercept and slope values a heat map of predictive probability was created to determine likelihood of GERD, EoE or non-GERD during endoscopy (Figure 4). The probability of EoE is highest with low MI values distally 
(intercept) in the esophagus and low slope (minimal rise of MI with distance away from SCJ) (shown in blue), while a greater rise in MI slope with low MI intercept distally is suggestive of GERD (shown in red), and higher MI values distally with higher slope is suggestive of non-GERD (shown in gray).

Balloon MI was well tolerated with only one adverse event in a patient with EoE showing small distal esophageal mucosal tear similar to that seen during endoscopic dilatation. This patient reported mild chest pain post-procedure and was discharged home uneventfully.

\section{DISCUSSION}

Current diagnostic tests for esophageal disorders lack accuracy, timeliness, and reliability in detecting changes in mucosal integrity from long-term inflammation related to injurious gastroduodenal contents or from immune-mediated disease. There is also significant overlap between clinical presentation of GERD and EoE with heartburn reported in 30-60\% of patients with EoE. ${ }^{27,28}$ The overlap in symptoms can make clinical separation between GERD and EoE challenging prior to diagnostic testing given both are frequently in the differential. Furthermore, EoE can be patchy disease ${ }^{29}$ and while there are general recommendations on where to obtain biopsies to diagnose EoE (proximal and distal esophagus), one study showed that only $24 \%$ of academic and $3 \%$ of community gastroenterologists follow consensus guidelines to diagnose EoE. ${ }^{30}$ This has also led to significant variability in diagnostic criteria used by studies for EoE in the literature ranging from 5 to 30 eosinophils/ $\mathrm{HpF}$ with large proportion of studies (35\%) not even stating their diagnostic criteria. ${ }^{31}$ Due to need for esophageal biopsy for diagnosis and multiple endoscopies to document treatment response, the estimated median cost associated with EoE is approximately $\$ 2,302 /$ year/patient. ${ }^{32}$ Levels of esophageal eosinophilia have also been shown to vary widely throughout locations in the esophagus and even within biopsies themselves. ${ }^{33,} 34$ Thus, there is no way to ensure that biopsies taken will not miss a diagnosis of EoE or inaccurately express disease activity. Overlap among clinical presentations between GERD and EoE along with inadequate reliability of endoscopic features and histopathological changes can make it challenging for a clinician to reliably choose the correct diagnostic test.

In this multi-center prospective study, we showed the clinical performance of an innovative balloon MI measurement device that is easily, timely and reliably able to distinguish patients with GERD, EoE, or non-GERD during endoscopy. This technology allows the clinician to obtain MI measurements on a $10 \mathrm{~cm}$ segment of the esophagus, which can increase diagnostic yield especially in patchy diseases such as EoE. Important observations from our study include the following: 1) the contour of esophageal mucosal integrity is unique in GERD and EoE compared to non-GERD patients; 2) intercept and slope (rate of rise of MI from distal to proximal esophagus) of balloon MI measurements are able to discriminate between the three groups; and 3) our model can instantly provide the likely diagnosis (EoE, GERD, or non-GERD) during endoscopy, which can help reduce unnecessary testing. For instance, in a patient with only $2 \%$ predicted probability of GERD using balloon MI, alternative diagnoses may be considered eliminating the need for additional tests potentially reducing the cost for patient management. Our technique is safe, simple, can be performed 
during endoscopy, and adds approximately 2 minutes to the procedure time. We have also previously shown that MI measurements correlate with histopathology (degree of spongiosis and eosinophilia $)^{2,35}$ and normalize with treatment. ${ }^{14}$ Improvement in clinical symptoms on the other hand have low accuracy in predicting endoscopic or histologic remission in patients with EoE. ${ }^{36}$ Thus, in patients with EoE, MI can be a surrogate marker of histology and be used to monitor treatment response during endoscopy instead of repeated esophageal biopsies. More recently, we have shown in a prospective blinded study that MI can predict EoE without histology with sensitivity of $100 \%$ and specificity of $96 \% .{ }^{20}$ This could result in significant cost savings for patients, as addition of biopsy with pathologic interpretation usually adds an average of $\$ 235$ per endoscopic procedure (based on 2009 Medicare rates). 37

Testing for GERD has primarily relied on detection of intraluminal acid or non-acid refluxate over 24 to 48 hour period, which has led to significant variability in sensitivity and specificity as they fail to account for day-to-day variability of reflux. ${ }^{9,38,39}$ Multiple studies have shown nearly $20 \%$ discordance rate between day 1 and day 2 of total esophageal acid exposure using wireless $\mathrm{pH}$ monitoring. ${ }^{40-42}$ On the other hand, transnasal probes lose precision as they are not consistently in contact with the esophageal mucosa (due to movement in the esophageal lumen) and we have previously shown that this results in increased variability of measurements compared to direct MI measurements during endoscopy ${ }^{43}$ This has led to poor reliability of current reflux diagnostic testing in being able to predict response to medical or surgical therapies. ${ }^{44}$ Thus, focus has now shifted towards identifying novel diagnostic parameters that can provide instant diagnosis, reduce patient discomfort, and detect alternations in esophagus mucosal integrity. Impedance baseline measurements have been shown to correlate with esophageal mucosal integrity status. ${ }^{45}$ Lower baseline impedance has been associated with increased esophageal acid exposure ${ }^{46}$ and symptom perception. ${ }^{47}$ Newer metrics such as nocturnal baseline impedance from analysis of the impedance-pH monitoring tracings have been shown to be lower in patients with GERD who responded to PPI therapy or anti-reflux surgery compared with those who did not respond. ${ }^{48,49}$ Thus, measures of mucosal integrity in GERD might be able to better diagnose and dictate treatment options in this challenging patient population. We have also shown that the pattern of mucosal impedance contour is similar between our internal and external cohorts from three different tertiary care academic medical centers for GERD and EoE indicating generalizability of the results.

Our novel balloon MI device is a significant improvement from the proof of concept single channel MI catheter utilizing the following changes: 1) Use of $180^{\circ}$ impedance sensors mounted on an inflated balloon, which allows precise opposition of the sensor to the mucosal lining (compared to the $360^{\circ}$ circumferential ring design of the single channel catheter) and reduces interference from intraluminal contents such as air and liquid;2) the single channel MI catheter had to be manually repositioned from one site to another along the esophagus, which can result in inter-provider variability as we have previously shown. ${ }^{21}$ This is reduced now with use of a stationary inflated balloon with sensors mounted at $1 \mathrm{~cm}$ intervals; and 3) inclusion of multiple radial and axial MI sensors on the $10 \mathrm{~cm}$ balloon, which allows for wider sampling of esophageal mucosal integrity. Our clinical model using the intercept and slope can be used instantly during endoscopy to predict probability of 
GERD, EoE, or non-GERD, which can help guide clinical decision making. We believe that use of a model to provide probability of the disease is a better diagnostic modality compared to using arbitrary cut-offs as cut-offs can frequently be misleading in a clinical setting. Studies have shown that many physicians misunderstand basic concepts of test accuracy ${ }^{50-52}$ and have significant difficulty with estimating post-test probability using sensitivity/specificity of a diagnostic test. ${ }^{53}$ Our model provides post-test probability after balloon MI measurements and can aid in augmenting the clinicians pre-test probability of the disease instantly during endoscopy.

In a recent prospective blinded study using MI to predict GERD, we showed only $76 \%$ concordance rate between MI and wireless $\mathrm{pH}$ monitoring, while MI had 100\% sensitivity and specificity for esophagitis. ${ }^{54}$ Long-term outcome studies are needed to see if changes in mucosal integrity detected by balloon MI are a better predictor for response to medical or surgical treatment in patients with GERD compared to ambulatory $\mathrm{pH}$ testing. However, balloon MI provides an instant diagnosis by evaluating esophageal epithelial mucosal integrity during endoscopy, while ambulatory $\mathrm{pH}$ testing can take multiple days for diagnosis and interpretation.

We do acknowledge that this study has some limitations. Our prediction model currently assumes that subjects must belong to one of the three diagnosis groups, and uses an equal baseline prevalence of GERD, non-GERD, and EoE (35\%, 35\%, 30\%) to estimate conditional (post-test) probability of the disease given MI intercept and slope. However, clinically, certain demographic and clinical symptoms can help us augment the pre-test probability. For instance, studies have shown that clinical features that independently predicted EoE were younger age ( $<50$ years), male, symptoms of dysphagia or history of food impaction, and documented food allergies/asthma. ${ }^{27,} 28 \mathrm{An}$ ideal clinical prediction model would incorporate clinical characteristics to change the pre-test probability of a disease and then use balloon MI to provide more definitive post-test probability of the disease. However, this would require a very large sample size and we are now in the process of performing future studies using clinical characteristics in addition to balloon MI to augment the prediction model. It should also be noted that use of balloon MI should be avoided in patients with severe fibrostenotic disease that precludes safe expansion of the balloon catheter. Our study also did not include patients with esophageal dysmotility.

In conclusion, we have developed a novel balloon mucosal impedance device for detecting esophageal mucosal changes due to chronic GERD or EoE instantly during routine endoscopy potentially obviating the need for 24 to 48 hour ambulatory wireless $\mathrm{pH}$ monitoring or esophageal biopsies for histopathology. This can help reduce diagnostic and treatment latency and might allow for monitoring disease activity over time.

\section{Supplementary Material}

Refer to Web version on PubMed Central for supplementary material.

\section{Funding:}

NIH NIDDK R01 DK092217 (JP, external validation data set). 


\section{REFERENCES}

1. Dent J, El-Serag HB, Wallander MA, et al. Epidemiology of gastro-oesophageal reflux disease: a systematic review. Gut 2005;54:710-7. [PubMed: 15831922]

2. Sontaj SJ. The medical management of reflux esophagitis. Role of antacids and acid inhibition. Gastroenterol Clin North Am 1990;19:683-712. [PubMed: 1977703]

3. El-Serag HB, Sweet S, Winchester CC, et al. Update on the epidemiology of gastro-oesophageal reflux disease: a systematic review. Gut 2014;63:871-80. [PubMed: 23853213]

4. Fenter TC, Naslund MJ, Shah MB, et al. The cost of treating the 10 most prevalent diseases in men 50 years of age or older. Am J Manag Care 2006;12:S90-8. [PubMed: 16551207]

5. Everhart JE, Ruhl CE. Burden of digestive diseases in the United States part I: overall and upper gastrointestinal diseases. Gastroenterology 2009;136:376-86. [PubMed: 19124023]

6. Fass R, Hell R, Sampliner RE, et al. Effect of ambulatory 24-hour esophageal pH monitoring on reflux-provoking activities. Dig Dis Sci 1999;44:2263-9. [PubMed: 10573372]

7. Pandolfino JE, Schreiner MA, Lee TJ, et al. Comparison of the Bravo wireless and Digitrapper catheter-based $\mathrm{pH}$ monitoring systems for measuring esophageal acid exposure. Am J Gastroenterol 2005;100:1466-76. [PubMed: 15984967]

8. Pandolfino JE, Richter JE, Ours T, et al. Ambulatory esophageal $\mathrm{pH}$ monitoring using a wireless system. Am J Gastroenterol 2003;98:740-9. [PubMed: 12738450]

9. Patel DA, Vaezi MF. Utility of esophageal mucosal impedance as a diagnostic test for esophageal disease. Curr Opin Gastroenterol 2017;33:277-284. [PubMed: 28437259]

10. Wenner J, Johansson J, Johnsson F, et al. Optimal thresholds and discriminatory power of 48-h wireless esophageal pH monitoring in the diagnosisof GERD. Am J Gastroenterol 2007;102:18629. [PubMed: 17509034]

11. Kessels SJM, Newton SS, Morona JK, et al. Safety and Efficacy of Wireless pH Monitoring in Patients Suspected of Gastroesophageal Reflux Disease: A Systematic Review. J Clin Gastroenterol 2017;51:777-788. [PubMed: 28877081]

12. Dellon ES, Jensen ET, Martin CF, et al. Prevalence of eosinophilic esophagitis in the United States. Clin Gastroenterol Hepatol 2014;12:589-96 e1. [PubMed: 24035773]

13. Furuta GT, Katzka DA. Eosinophilic Esophagitis. N Engl J Med 2015;373:1640-8. [PubMed: 26488694]

14. Ates F, Yuksel ES, Higginbotham T, et al. Mucosal impedance discriminates GERD from nonGERD conditions. Gastroenterology 2015;148:334-43. [PubMed: 25448923]

15. Tobey NA, Carson JL, Alkiek RA, et al. Dilated intercellular spaces: a morphological feature of acid reflux--damaged human esophageal epithelium. Gastroenterology 1996;111:1200-5. [PubMed: 8898633]

16. Caviglia R, Ribolsi M, Maggiano N, et al. Dilated intercellular spaces of esophageal epithelium in nonerosive reflux disease patients with physiological esophageal acid exposure. Am J Gastroenterol 2005;100:543-8. [PubMed: 15743349]

17. Katzka DA, Tadi R, Smyrk TC, et al. Effects of topical steroids on tight junction proteins and spongiosis in esophageal epithelia of patients with eosinophilic esophagitis. Clin Gastroenterol Hepatol 2014;12:1824-9 e1. [PubMed: 24681080]

18. Katzka DA, Ravi K, Geno DM, et al. Endoscopic Mucosal Impedance Measurements Correlate With Eosinophilia and Dilation of Intercellular Spaces in Patients With Eosinophilic Esophagitis. Clin Gastroenterol Hepatol 2015;13:1242-1248 e1. [PubMed: 25592662]

19. Lowry MA, Vaezi M, Correa H, et al. 704 An Innovative Mucosal Impedance Device Differentiates Active Eosinophilic Esophagitis From Inactive Disease, NERD and Controls. Gastrointestinal Endoscopy;83:AB166.

20. Choksi Y, Lal P, Slaughter JC, et al. Esophageal Mucosal Impedance Patterns Discriminate Patients With Eosinophilic Esophagitis From Patients With GERD. Clin Gastroenterol Hepatol 2018;16:664-671 e1. [PubMed: 29248733]

21. Sharda R, Choksi YA, Higginbotham T, et al. Sa1294 Reducing Mucosal Impedance Variability by Ensuring Direct Contact with Esophageal Mucosa. Gastroenterology 2016;150:S272. 
22. Lundell LR, Dent J, Bennett JR, et al. Endoscopic assessment of oesophagitis: clinical and functional correlates and further validation of the Los Angeles classification. Gut 1999;45:172-80. [PubMed: 10403727]

23. Dellon ES, Gonsalves N, Hirano I, et al. ACG Clinical Guideline: Evidenced Based Approach to the Diagnosis and Management of Esophageal Eosinophilia and Eosinophilic Esophagitis (EoE). The American Journal Of Gastroenterology 2013;108:679. [PubMed: 23567357]

24. Patel DA, Wright MR, Higginbotham T, et al. Novel Balloon Mucosal Impedance for Diagnosis of Gerd and EOE. Gastroenterology 2017;152:S43.

25. Pritchett JM, Aslam M, Slaughter JC, et al. Efficacy of esophageal impedance/pH monitoring in patients with refractory gastroesophageal reflux disease, on and off therapy. Clin Gastroenterol Hepatol 2009;7:743-8. [PubMed: 19281866]

26. Kavitt RT, Yuksel ES, Slaughter JC, et al. The role of impedance monitoring in patients with extraesophageal symptoms. Laryngoscope 2013;123:2463-8. [PubMed: 23857711]

27. Dellon ES, Gibbs WB, Fritchie KJ, et al. Clinical, endoscopic, and histologic findings distinguish eosinophilic esophagitis from gastroesophageal reflux disease. Clin Gastroenterol Hepatol 2009;7:1305-13; quiz 1261. [PubMed: 19733260]

28. Veerappan GR, Perry JL, Duncan TJ, et al. Prevalence of eosinophilic esophagitis in an adult population undergoing upper endoscopy: a prospective study. Clin Gastroenterol Hepatol 2009;7:420-6, 426 e1-2. [PubMed: 19162236]

29. Odze RD. Pathology of Eosinophilic Esophagitis: What the Clinician Needs to Know. The American Journal Of Gastroenterology 2009;104:485. [PubMed: 19174804]

30. Peery AF, Shaheen NJ, Dellon ES. Practice patterns for the evaluation and treatment of eosinophilic oesophagitis. Aliment Pharmacol Ther 2010;32:1373-82. [PubMed: 21050240]

31. Dellon ES, Aderoju A, Woosley JT, et al. Variability in diagnostic criteria for eosinophilic esophagitis: a systematic review. Am J Gastroenterol 2007;102:2300-13. [PubMed: 17617209]

32. Jensen ET, Kappelman MD, Martin CF, et al. Health-care utilization, costs, and the burden of disease related to eosinophilic esophagitis in the United States. Am J Gastroenterol 2015;110:62632. [PubMed: 25267327]

33. Saffari H, Peterson KA, Fang JC, et al. Patchy eosinophil distributions in an esophagectomy specimen from a patient with eosinophilic esophagitis: Implications for endoscopic biopsy. $\mathbf{J}$ Allergy Clin Immunol 2012;130:798-800. [PubMed: 22502795]

34. Gonsalves N, Policarpio-Nicolas M, Zhang Q, et al. Histopathologic variability and endoscopic correlates in adults with eosinophilic esophagitis. Gastrointest Endosc 2006;64:313-9. [PubMed: 16923475]

35. Lowry MA, Vaezi MF, Correa H, et al. Mucosal Impedance Measurements Differentiate Pediatric Patients with Active vs Inactive Eosinophilic Esophagitis. J Pediatr Gastroenterol Nutr 2018.

36. Safroneeva E, Straumann A, Coslovsky M, et al. Symptoms Have Modest Accuracy in Detecting Endoscopic and Histologic Remission in Adults With Eosinophilic Esophagitis. Gastroenterology 2016;150:581-590 e4. [PubMed: 26584601]

37. Miller SM, Goldstein JL, Gerson LB. Cost-effectiveness model of endoscopic biopsy for eosinophilic esophagitis in patients with refractory GERD. Am J Gastroenterol 2011;106:1439-45. [PubMed: 21448144]

38. Richter JE. How to manage refractory GERD. Nature Clinical Practice Gastroenterology \&Amp; Hepatology 2007;4:658.

39. Fass R, Gasiorowska A. Refractory GERD: what is it? Curr Gastroenterol Rep 2008;10:252-7. [PubMed: 18625135]

40. Turner BG, Saltzman JR, Hua L, et al. Endoscopic pH monitoring for patients with suspected or refractory gastroesophageal reflux disease. Can J Gastroenterol 2007;21:737-41. [PubMed: 18026578]

41. Ahlawat SK, Novak DJ, Williams DC, et al. Day-to-day variability in acid reflux patterns using the BRAVO pH monitoring system. J Clin Gastroenterol 2006;40:20-4. [PubMed: 16340628]

42. Ayazi S, Hagen JA, Zehetner J, et al. Day-to-day discrepancy in Bravo pH monitoring is related to the degree of deterioration of the lower esophageal sphincter and severity of reflux disease. Surg Endosc 2011;25:2219-23. [PubMed: 21359906] 
43. Kim HP, Heller LT, Ates F, et al. Mo1132 Intraluminal Impedance Versus Mucosal Impedance Testing for the Diagnosis of GERD: Do They Measure the Same Thing? Gastroenterology 2015; 148:S-615-S-616.

44. Vaezi MF, Sifrim D. Assessing Old and New Diagnostic Tests for Gastroesophageal Reflux Disease. Gastroenterology 2018;154:289-301. [PubMed: 28774844]

45. Farre R, Blondeau K, Clement D, et al. Evaluation of oesophageal mucosa integrity by the intraluminal impedance technique. Gut 2011;60:885-92. [PubMed: 21303918]

46. Kessing BF, Bredenoord AJ, Weijenborg PW, et al. Esophageal acid exposure decreases intraluminal baseline impedance levels. Am J Gastroenterol 2011;106:2093-7. [PubMed: 21844921]

47. Woodland P, Al-Zinaty M, Yazaki E, et al. In vivo evaluation of acid-induced changes in oesophageal mucosa integrity and sensitivity in non-erosive reflux disease. Gut 2013;62:1256-61. [PubMed: 22722617]

48. de Bortoli N, Martinucci I, Savarino E, et al. Association between baseline impedance values and response proton pump inhibitors in patients with heartburn. Clin Gastroenterol Hepatol 2015;13:1082-8 e1. [PubMed: 25499991]

49. Patel A, Wang D, Sainani N, et al. Distal mean nocturnal baseline impedance on $\mathrm{pH}$-impedance monitoring predicts reflux burden and symptomatic outcome in gastro-oesophageal reflux disease. Aliment Pharmacol Ther 2016;44:890-8. [PubMed: 27554638]

50. Lyman GH, Balducci L. The effect of changing disease risk on clinical reasoning. J Gen Intern Med 1994;9:488-95. [PubMed: 7996291]

51. Puhan MA, Steurer J, Bachmann LM, et al. A randomized trial of ways to describe test accuracy: the effect on physicians' post-test probability estimates. Ann Intern Med 2005;143:184-9. [PubMed: 16061916]

52. Sox CM, Doctor JN, Koepsell TD, et al. The influence of types of decision support on physicians' decision making. Arch Dis Child 2009;94:185-90. [PubMed: 19131417]

53. Agoritsas T, Courvoisier DS, Combescure C, et al. Does prevalence matter to physicians in estimating post-test probability of disease? A randomized trial. J Gen Intern Med 2011;26:373-8. [PubMed: 21053091]

54. Patel DA, Bennett A, Sarker S, et al. Su1084 - Concordance Between Mucosal Impedance Measurements at Index Endoscopy and Wireless PH Monitoring: A Prospective Blinded Study. Gastroenterology 2018;154:S-481-S-482. 


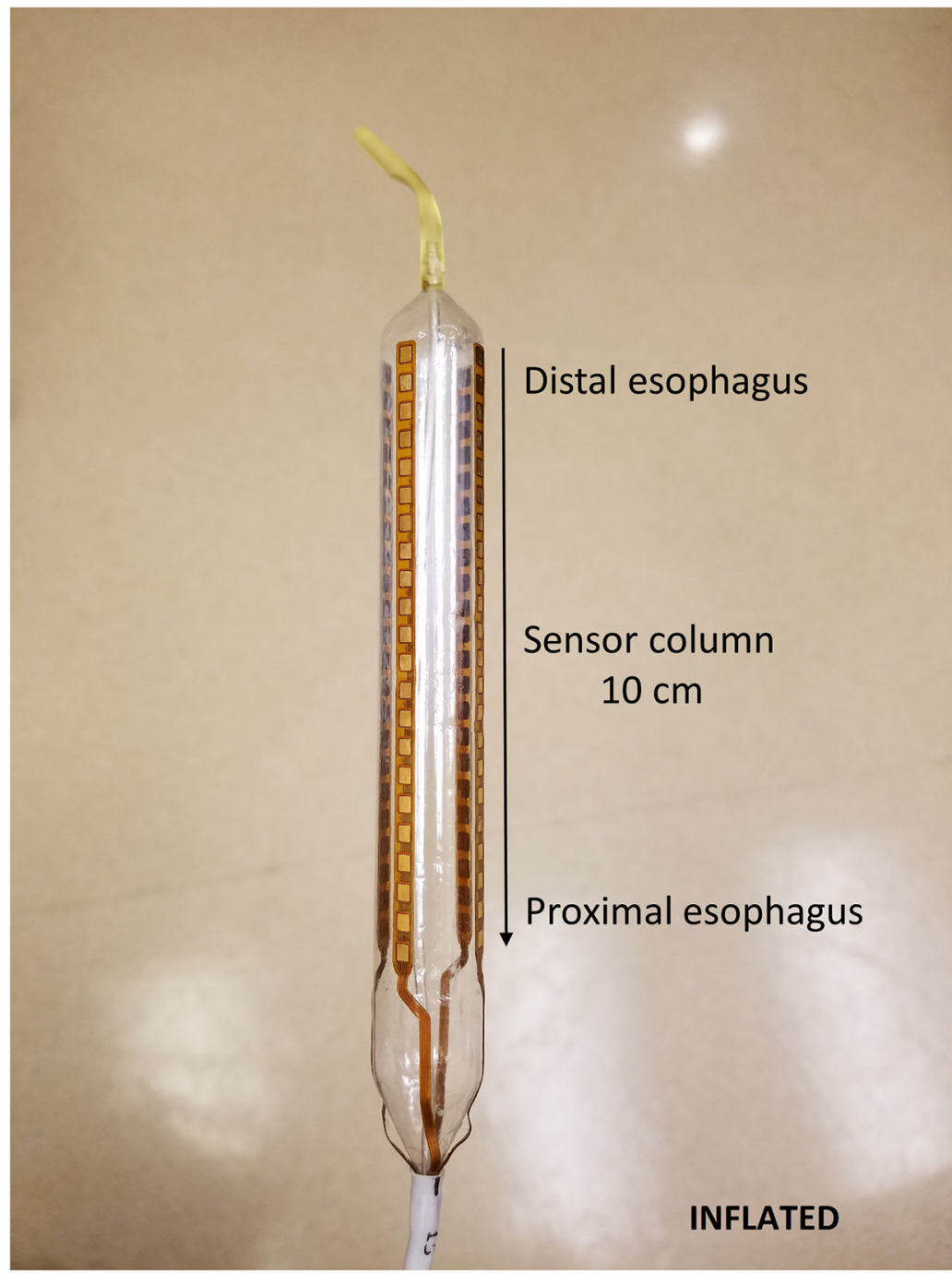

Figure 1.

Novel MI balloon catheter with 36 channels measuring impedance axially and radially along a $10 \mathrm{~cm}$ length of the esophagus. 

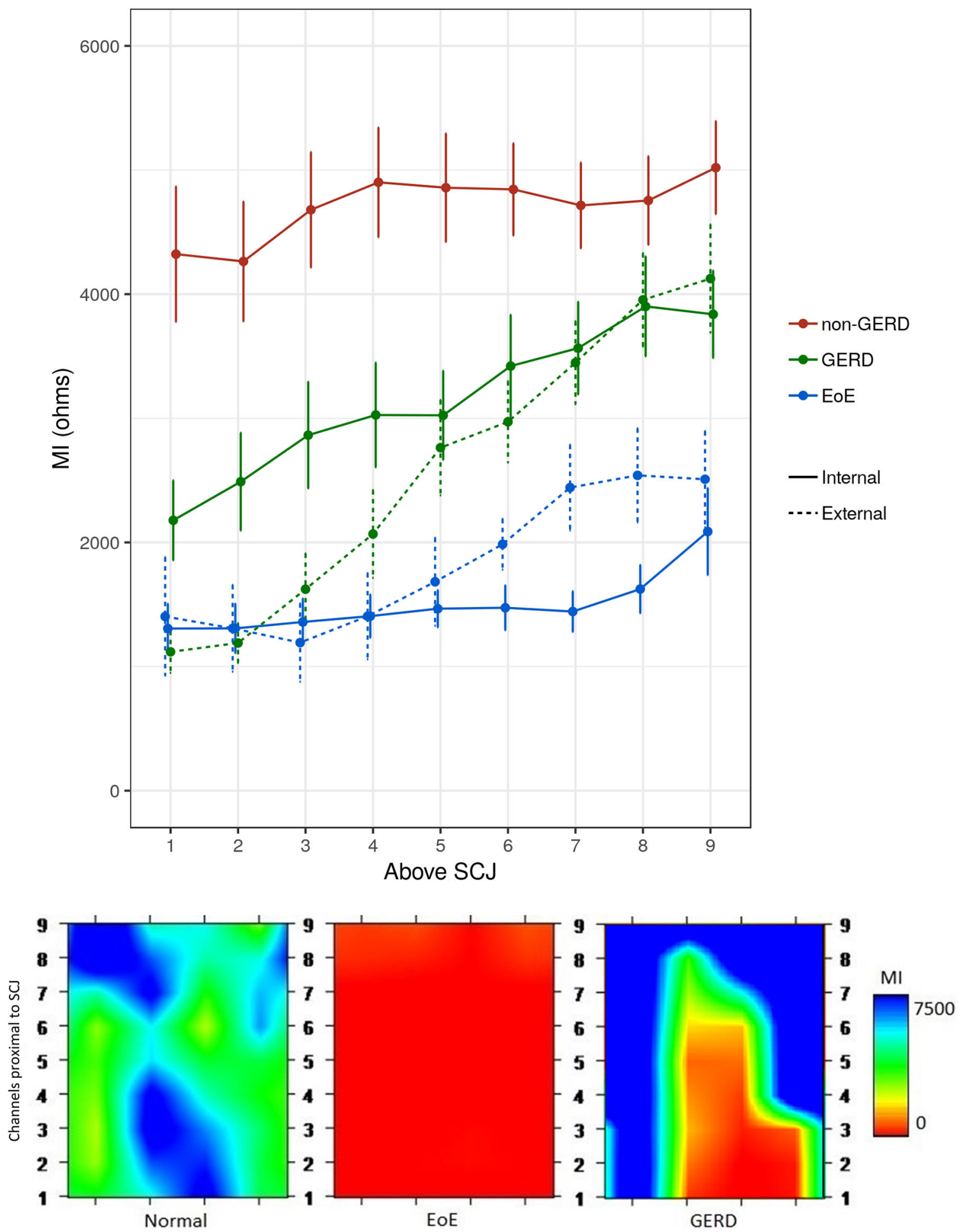

Figure 2.

A) Average MI and standard error over channels from distal to proximal esophagus (1-> 9) by diagnosis group in both internal (solid line) and external cohorts (dotted line). B) MI measurements $(\Omega)$ stratified by esophageal pathology and distance from SCJ. X-axis represents columns of impedance sensors 90 degrees apart. B) Loss of esophageal mucosal 
integrity results in low MI values color coded as red (seen distally in both GERD and EoE), but the MI values remained low in EoE proximally (due to pan-esophageal involvement), while it normalizes in GERD (green/yellow). Non-GERD patients have no disruption in mucosal integrity resulting in normal MI values (green and blue). 


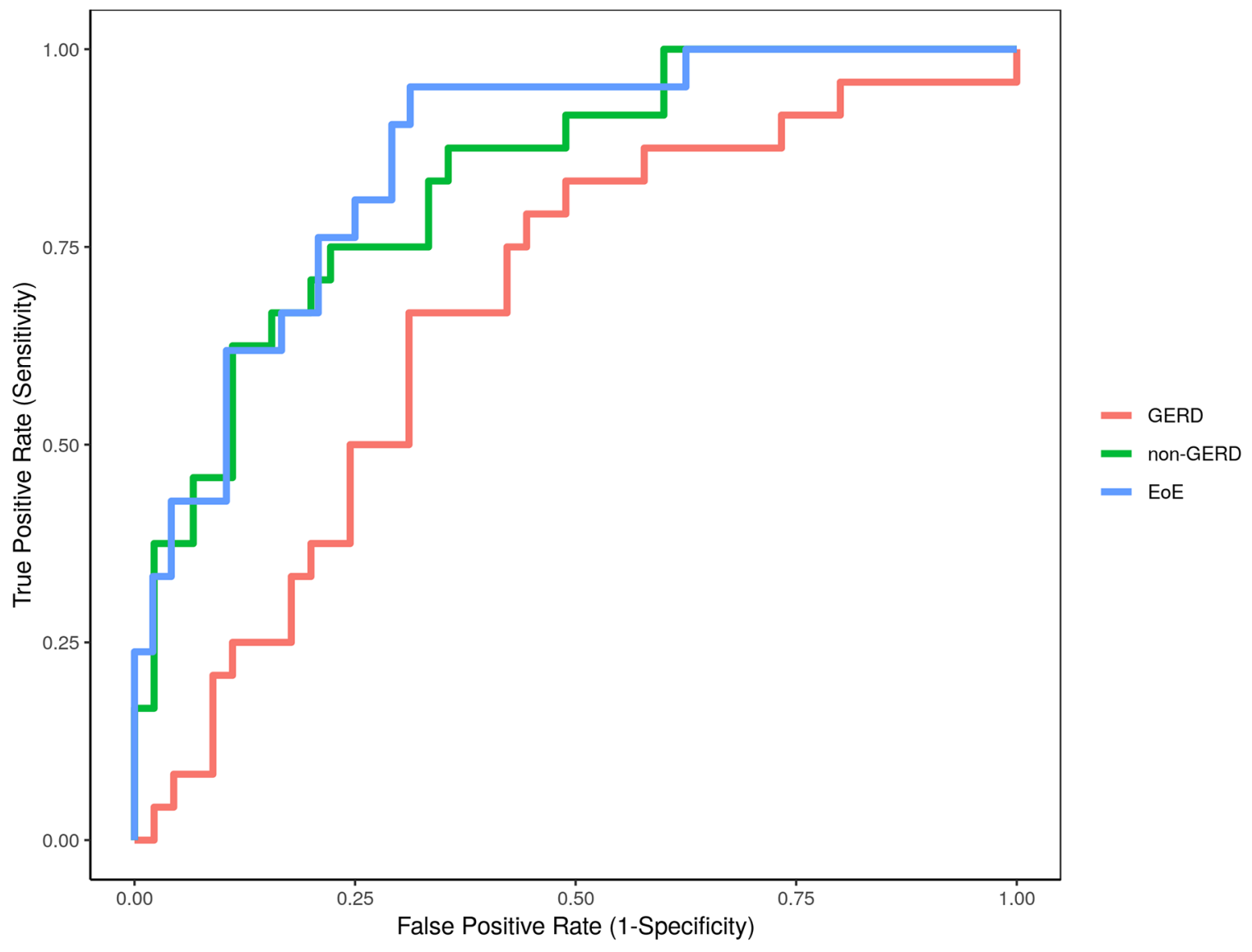

Figure 3.

Receiver operating characteristic analysis of intercept and slope of balloon MI measurements along the esophageal axis for diagnosis of GERD, EoE, and non-GERD. 


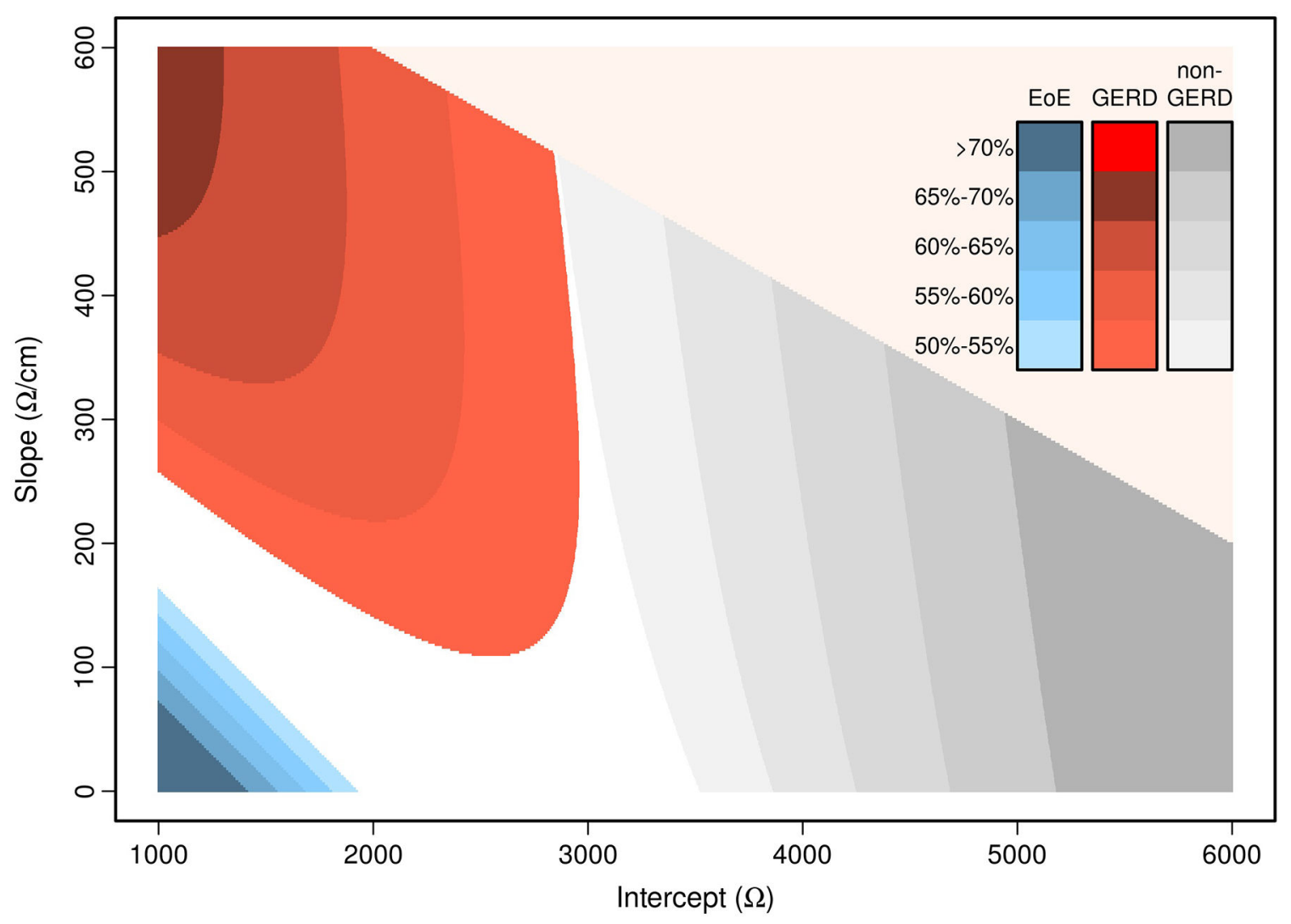

Figure 4.

Predicted probabilities of GERD, non-GERD, or EoE stratified by MI intercept and slope (rate of rise from distal to proximal esophagus). Shades of colors show relative probabilities of the condition (Blue for EoE; Red for GERD; and Gray for non-GERD). Low intercept (measurement at the most distal channel) and low slope (rise of MI from distal to proximal esophagus) is suggestive of $\mathrm{EoE}$ (blue), while low intercept, but greater rise in slope is suggestive of GERD (red). 
Table 1:

Baseline demographics of patients.

\begin{tabular}{|c|c|c|c|c|}
\hline & GERD $(\mathrm{N}=24)$ & Non-GERD $(\mathrm{N}=\mathbf{2 4})$ & $\operatorname{EoE}(\mathbf{N}=\mathbf{2 1})$ & p-value \\
\hline Age & $48(42-62)$ & $62(50-67)$ & $33(26-38)$ & $<0.01^{2}$ \\
\hline BMI & $30(27-35)$ & $27(25-35)$ & $27(25-28)$ & $0.09^{2}$ \\
\hline Female, $\%(\mathrm{n})$ & $54 \%$ & $71 \%$ & $33 \%$ & $0.07^{1}$ \\
\hline Ethnicity, \% (n) & & & & $0.54^{1}$ \\
\hline Caucasian & $92 \%$ & $92 \%$ & $100 \%$ & \\
\hline Black & $4 \%$ & $8 \%$ & $0 \%$ & \\
\hline Other & $4 \%$ & $0 \%$ & $0 \%$ & \\
\hline Chief Complaint, \% (n) & & & & $<0.01^{1}$ \\
\hline GERD (heartburn or regurgitation) & $63 \%$ & $66 \%$ & $7 \%$ & \\
\hline Dysphagia & $12 \%$ & $8 \%$ & $93 \%$ & \\
\hline Other (atypical symptoms) & $25 \%$ & $25 \%$ & $0 \%$ & \\
\hline Presence of esophagitis, $\%$ (n) & $83 \%$ & & & \\
\hline $\mathrm{A} / \mathrm{B}$ & $60 \%$ & & & \\
\hline $\mathrm{C} / \mathrm{D}$ & $40 \%$ & & & \\
\hline Presence of hiatal hernia, \% (n) & $27 \%$ & $15 \%$ & $0 \%$ & $0.14^{1}$ \\
\hline Size of hiatal hernia $(\mathrm{cm})$ & $3.5(2-5.2)$ & $3.0(2-4.5)$ & & $0.88^{3}$ \\
\hline \multicolumn{5}{|l|}{$\mathrm{pH}$ parameters } \\
\hline Total $\%$ time $\mathrm{pH}<4$ & $11.2(9.6-14.7)$ & $2.7(1.0-3.8)$ & & $<0.01^{3}$ \\
\hline Upright $\%$ time $\mathrm{pH}<4$ & $14.8(10.6-18.4)$ & $3.0(1.3-5.2)$ & & $<0.01^{3}$ \\
\hline Supine $\%$ time $\mathrm{pH}<4$ & $3.9(2.4-6.0)$ & $0.1(0-0.7)$ & & $<0.01^{3}$ \\
\hline \multicolumn{5}{|l|}{ Eosinophilic count (per HpF) } \\
\hline Proximal esophagus & & & $50(30-100)$ & \\
\hline Distal esophagus & & & $47(40-50)$ & \\
\hline
\end{tabular}

The ranges in parentheses for age, BMI, eosinophilic count, and Total/upright/supine $\%$ time $\mathrm{pH}<4$ represent the interquartile range.

Test used:

Pearson test;

2 Kruskal-Wallis test;

3 Wilcoxon test.

HpF: High power field. 
Table 2:

Median (IQR) MI values (in Ohms) along esophageal axis in each group.

\begin{tabular}{|c|c|c|c|c|}
\hline Channels above squamocolumnar junction $^{*}$ & GERD $(\mathbf{N}=\mathbf{2 4})$ & Non-GERD $(\mathbf{N}=\mathbf{2 4})$ & EoE $(\mathbf{N = 2 1})$ & p- value \\
\hline 1 & $1668(971-2754)$ & $4198(1911-6475)$ & $1046(842-1896)$ & $<0.01$ \\
\hline 2 & $2077(1041-3020)$ & $4062(2107-6036)$ & $1053(789-1727)$ & $<0.01$ \\
\hline 3 & $1713(1265-4056)$ & $4473(2752-6460)$ & $1203(774-1816)$ & $<0.01$ \\
\hline 4 & $2263(1261-4763)$ & $5501(3087-6643)$ & $1493(938-2052)$ & $<0.01$ \\
\hline 5 & $2563(1660-3686)$ & $4847(3159-6566)$ & $1539(935-1955)$ & $<0.01$ \\
\hline 6 & $3103(2346-4091)$ & $5215(3737-5927)$ & $1706(1013-2105)$ & $<0.01$ \\
\hline 7 & $3226(2331-4740)$ & $5000(3940-5647)$ & $1813(1003-2594)$ & $<0.01$ \\
\hline 8 & $3595(2637-4917)$ & $4592(4181-6059)$ & $2106(1018-2647)$ & $<0.01$ \\
\hline 9 & $4190(2444-4948)$ & $5089(3986-6176)$ & $2371(1268-3431)$ & $<0.01$ \\
\hline Intercept & $1490(653-3475)$ & $4095(1990-6583)$ & $785(501-1343)$ & $<0.01$ \\
\hline Slope & $242(115,323)$ & $68(-84,186)$ & $-12(-80,263)$ & 0.04 \\
\hline
\end{tabular}

* Each sensor is separated by 1 -cm with channel 1 being just above the SCJ and channel 9 in proximal esophagus. IQR represent the interquartile range. Test used: Kruskal-Wallis test. 
Table 3:

Comparison of predictive accuracy of different models.

\begin{tabular}{|l|l|c|c|c|c|}
\hline \multirow{3}{*}{ EoE } & Models & C-index & Brier Score & $\mathbf{R}^{\mathbf{2}}$ & AIC \\
\cline { 2 - 6 } & MI at 2 and $5 \mathrm{~cm}$ & 0.89 & 0.13 & 0.52 & 57.8 \\
\cline { 2 - 6 } & MI at 2 and $10 \mathrm{~cm}$ & 0.86 & 0.14 & 0.49 & 59.7 \\
\cline { 2 - 6 } & Intercept and slope & 0.89 & 0.12 & 0.55 & 54.9 \\
\hline \multirow{3}{*}{ GERD } & MI at 2 and $5 \mathrm{~cm}$ & 0.71 & 0.21 & 0.10 & 89.0 \\
\cline { 2 - 6 } & MI at 2 and $10 \mathrm{~cm}$ & 0.54 & 0.23 & 0.01 & 93.7 \\
\cline { 2 - 6 } & Intercept and slope & 0.69 & 0.21 & 0.11 & 88.5 \\
\hline \multirow{3}{*}{ Non-GERD } & MI at 2 and $5 \mathrm{~cm}$ & 0.81 & 0.17 & 0.33 & 75.8 \\
\cline { 2 - 6 } & MI at 2 and $10 \mathrm{~cm}$ & 0.84 & 0.15 & 0.41 & 70.3 \\
\cline { 2 - 6 } & Intercept and slope & 0.84 & 0.16 & 0.40 & 70.9 \\
\hline
\end{tabular}

$\mathrm{R}^{2}$ (correlation coefficient); AIC (Akaike information criterion). 